\title{
Topos Theoretic Quantum Realism
}

\author{
Benjamin Eva
}

(Forthcoming in British Journal for the Philosophy of Science)

\begin{abstract}
Topos Quantum Theory $(\mathrm{TQT})^{1}$ is standardly portrayed as a kind of 'neo-realist' reformulation of quantum mechanics. In this paper, we study the extent to which TQT can really be characterised as a realist formulation of the theory, and examine the question of whether the kind of realism that is provided by TQT satisfies the philosophical motivations that are usually associated with the search for a realist reformulation of quantum theory. Specifically, we show that the notion of the quantum state is problematic for those who view TQT as a realist reformulation of quantum theory.
\end{abstract}

1 Introduction

2 Topos Quantum Theory (TQT)

2.1 Phase space

2.2 Hilbert space

2.3 Beyond Hilbert space

2.4 Defining realism

2.5 The spectral presheaf

2.6 The logic of TQT

3 Interpreting States in $T Q T$

4 Interpreting Truth Values and Clopen Subobjects in TQT

4.1 Interpreting the truth values

4.2 Interpreting $S u b_{c l}(\underline{\Sigma})$

5 Neo-Realism

5.1 The covariant approach

6 Conclusion

\section{Introduction}

The topos theoretic reformulation of quantum theory (TQT) was originally proposed by Isham ([1997]), and has subsequently been developed

\footnotetext{
${ }^{1}$ I use the name 'topos quantum theory' to refer to the topos theoretic approach to quantum theory pioneered by Chris Isham, Jeremy Butterfield and Andreas Döring, which is often referred to as 'the contravariant approach' in the relevant literature. Although various other contemporaneous projects (some of which are discussed in section 5 of this paper) have applied topos theory to the study of quantum mechanics, the name 'topos quantum theory' appears to have been claimed by the contravariant approach (see Flori [2013]), and I follow this convention here.
} 
by Isham, Butterfield and Döring amongst others (Isham and Butterfield [1998], Döring [2012], Döring and Isham [2011]). From its inception, TQT has been motivated, at least in part, by broadly philosophical considerations. Specifically, TQT is often characterized as an attempt to replace the traditional 'instrumentalist' formulation of quantum mechanics (Hilbert space) with a new formalism that is more susceptible to a 'realist' interpretation. In this paper, we study the extent to which TQT, under such a characterization, has been successful as a philosophical project.

In section 2 , we begin by providing a concise technical overview of some of the central concepts and results of TQT, together with a summary of the key philosophical motivations of the project, and a discussion of the philosophical merits of the definition of realism that is usually employed in this context.

Each of the following sections focuses on one central aspect of the quantum realism provided by TQT. Section 3 focuses on the notion of the quantum state in TQT, and its relationship to the corresponding notion in the traditional Hilbert space formalism. In particular, we show that the identification of the spectral presheaf as the quantum state space is unmotivated and problematic

Section 4 provides an analysis of the logical structure of TQT. Specifically, it examines the physical significance of the truth values used in the theory, and provides a comparison with other attempts to use many valued logics in quantum theory, and then raises a significant interpretational issue concerning the lattice of physical propositions in TQT. Section 5 combines the analyses of the previous sections to provide a general appraisal of the quantum realism of TQT, and gives a brief discussion of the applicability of the arguments made in this paper to the topos theoretic approach to quantum theory developed, for example, in (Heunen et al [2009]). Section 6 concludes.

\section{Topos Quantum Theory}

We begin this section with a brief summary of the traditional formalisms of classical and quantum physics, and the asymmetries between them.

\subsection{Phase space}

The canonical formalism of classical physics can be summarized in the following way.

Let $S$ be a classical physical system. Then, to $S$ we associate a set $\mathbf{S}$ (the 'state space of $S$ ') consisting of the set of all possible states of the system $S$. To each physical quantity $A$ associated with $S$ (e.g momentum, position etc), we define a unique corresponding function $f_{A}: \mathbf{S} \rightarrow \mathbb{R}$. Given $s \in \mathbf{S}$, we interpret the value $f_{A}(s)$ as the value that the physical quantity $A$ would have if the state of $S$ were $s$.

Next, we define the notion of a physical proposition associated with $S$. Specifically, if $A$ is any physical quantity associated with $S$ and $\Delta$ is any Borel subset of the real numbers, we call the sentence 'the value of $A$ lies in the set $\Delta^{\prime}$ (denoted $\left.A \in \Delta\right)$ a 'physical proposition associated 
with $S^{\prime}$. Mathematically, we represent a physical proposition $A \in \Delta$ as the inverse image of $\Delta$ under $f_{A}$. Generalizing, we can identify physical propositions about $S$ as the inverse images of Borel subsets of the reals under real-valued functions on $\mathbf{S}$, i.e. physical propositions about $S$ are just measurable subsets of $\mathbf{S}$. Ordered by inclusion, the measurable subsets of $\mathbf{S}$ form a Boolean algebra, $S u b(\mathbf{S})$.

Now, let $s \in \mathbf{S}$. Then $s$ automatically defines a way of assigning classical truth values to all of the propositions associated with $S$. Specifically, given a physical proposition $P=A \in \Delta$, we define the truth value of $P$ relative to $S$ to be 1 if $s \in P=f_{A}^{-1}(\Delta)$ and 0 otherwise. It is easy to see that this assignment defines a Boolean algebra homomorphism, $h_{s}: \operatorname{Sub}(\mathbf{S}) \rightarrow\{0,1\}$ from the set of all physical propositions associated with $S$ to the two-element Boolean algebra.

To summarize, the canonical formalism of classical physics has two relevant fundamental properties. Firstly, the algebra of propositions associated with a classical system forms a Boolean algebra in a natural way. Secondly, any state of a classical physical system automatically defines a Boolean algebra homomorphism between the algebra of physical propositions associated with that system and the two element truth value algebra.

\section{$2.2 \quad$ Hilbert space}

In contrast to the canonical formalism for classical physics, the traditional Hilbert space formalism of quantum theory begins by assigning a complex Hilbert space $H$ to the physical system $S$ under consideration. The unit vectors of $H$ are then taken to represent the possible states of $S$. Any physical quantity $A$ associated with the system is then represented by a self-adjoint operator $\hat{A}$ on $H$. Then, given an eigenvector $|\psi\rangle$ of $\hat{A},|\psi\rangle$ 's eigenvalue with respect to $\hat{A}$ is interpreted as the value that $A$ would have if the state of $S$ were $|\psi\rangle$.

A physical proposition $A \in \Delta$ is then represented by the projection operator corresponding to the closed linear subspace consisting of those eigenvectors of $\hat{A}$ with eigenvalues in $\Delta$. Generalizing, we think of all closed linear subspaces (or, equivalently, all projection operators) of $H$ as representing physical propositions about $S$. It is well known that the lattice of projection operators $P(H)$ onto $H$ will generally fail to form a Boolean algebra and will only form an orthomodular lattice. This is in stark contrast to the formalism of classical physics, where the algebra of propositions about $S$ is always Boolean.

We have seen that in classical physics, a physical state of a system $S$ automatically defines a consistent assignment of classical truth values to all of the physical propositions associated with $S$. This is not the case in the Hilbert space formalism of quantum theory, where states will only, in general, provide probabilities for the truth/falsity of any given proposition. Indeed, it turns out that the existence of a consistent assignment of classical truth values to all of the physical propositions associated with a quantum system is generally impossible.

Kochen-Specker Theorem (KST): Let $H$ be a Hilbert space with 
dimension $\operatorname{dim}(H) \geq 3$. Then there is no way of assigning classical truth values to all of the projections operators on $H$ in a way that preserves the functional relationships ${ }^{2}$ between those projection operators.

Given the formalism outlined above, where physical propositions are identified with projection operators and physical quantities are identified with self-adjoint operators, KST can be interpreted as saying that it is impossible to simultaneously assign classical truth values to all of the propositions associated with a quantum system in a way that preserves the functional relationships between them, or equivalently, that it is impossible to assign definite values to all of the physical quantities associated with a quantum system in a way that preserves the functional relationships between them.

Again, this is radically disanalogous with the formalism of classical physics, where it is always possible, in principle, to assign classical truth values to all of the propositions associated with a physical system in a way that respects all of the algebraic relationships between those propositions.

\subsection{Beyond Hilbert space}

The fundamental motivating precept behind TQT is nicely summarized in the following passage:

'When dealing with a closed system, what is needed is a realist interpretation of the theory, not one that is instrumentalist. The exact meaning of "realist" is infinitely debatable, but, when used by physicists, it typically means the following:

(1). The idea of "a property of the system" (i.e. "the value of a physical quantity") is meaningful, and representable in the theory.

(2). Propositions about the system are handled using Boolean logic. This requirement is compelling in so far as we humans think in a Boolean way.

(3). There is a space of "microstates" such that specifying a microstate leads to unequivocal truth values for all propositions about the system. The existence of such a state space is a natural way of ensuring that the first two requirements are satisfied.

The standard interpretation of classical physics satisfies these requirements, and provides the paradigmatic example of a realist philosophy in science. On the other hand, the existence of such an interpretation in quantum theory is foiled by the famous Kochen-Specker theorem'. (Döring and Isham [2008])

The primary motivating problem for $\mathrm{TQT}^{3}$ is that, unlike classical physics, quantum physics is not amenable to a realist interpre-

\footnotetext{
${ }^{2}$ By 'functional relationships', we will mean the famous non-contextuality condition FUNC, which states that for any real valued function $f: \mathbb{R} \rightarrow \mathbb{R}$ and any assignment $V$ of classical truth values to projection operators, we should have $V(f(P))=f(V(P))$, where $P$ is any projection operator.

${ }^{3}$ At this point it should be noted that TQT has tended to focus primarily on the interpretational problems that occur even in synchronic contexts, and has been largely unconcerned
} 
tation, in the sense defined above. TQT can be construed as the attempt to provide a new formalism for quantum mechanics that, like the formalism of classical physics, has an unproblematically realist interpretation. The main obstacle to the availability of such a realist interpretation is KST. For, as we have seen, KST rules out the existence of a quantum state space satisfying condition (3).

By citing classical physics as the 'paradigmatic example' of a realist physical theory, Isham and Doering implicitly articulate one of the key methodological principles of TQT: to provide a formalism for quantum theory that, as far as possible, mimics and parallels the formalism of classical physics, and in doing so, inherits its archetypally realist properties.

Now, we have already had occasion to note two fundamental disanalogies between the formalisms of classical physics and quantum mechanics. Firstly, the algebra of propositions associated with a classical system, unlike the algebra of propositions associated with a quantum system, is Boolean. Secondly, a classical state, unlike a quantum state, automatically assigns classical truth values to all of the propositions associated with the system to which it corresponds. Indeed, classical states can actually be thought of as nothing more than Boolean algebra homomorphisms between the algebra of measurable subsets of the state space and the two element Boolean algebra. KST essentially tells us that such homomorphisms never exist for the algebra of physical propositions associated with a quantum system. As Bub puts it,

'while a classical property state is selected by a two-valued homomorphism on the Boolean lattice of classical properties, a quantum property state ... is not selected by a two-valued homomorphism on the non-Boolean lattice of subspaces representing the properties of a quantum mechanical system. There are no such two-valued homomorphisms'. (Bub [1999])

\subsection{Defining realism}

Isham and Döring's definition of realist physical theories employs three closely related criteria. We will consider each in turn.

The first criterion, that "the idea of "a property of the system" (i.e. "the value of a physical quantity") is meaningful, and representable in the theory' will henceforth be referred to as 'PROP'. Of course, PROP is a very natural condition to employ in a definition of what it means for a physical theory to be realist. For, if a purportedly realist theory allows us to talk about some physical quantity $Q$ associated with a physical system $S$, then of course

with the dynamics of quantum theory. Specifically, it has not yet been argued that TQT addresses the measurement problem. 
that theory should allow us to talk about $S$ having a value for $Q$, since how else could we conceive of $Q$ as really representing a feature of physical reality? One of the central interpretational problems of quantum theory, namely the problem of what one should say about those physical quantities that are represented by self-adjoint operators for which the state of the system is not an eigenstate, can be characterised as the problem of reconciling quantum theory with PROP.

The second criterion imposes the condition that in any realist physical theory, 'Propositions about the system are handled using Boolean logic'. We will refer to this condition as 'BOOLE'. BOOLE is really just an application to physics of Michael Dummett's principle that whenever one is realist about the subjects of a particular domain of discourse, they are thereby committed to using classical logic when reasoning in that domain (see e.g chapter 10 of Dummett [1978] ). This kind of position is often motivated by the idea that the law of excluded middle encodes a form of realism, in so far as it requires that the disjunction of any proposition with its own negation must be true. The idea is that the law of excluded middle prevents the world from being indifferent to the truth of any given proposition, since it requires that either the proposition or its negation must be true. In contrast, if a physical theory allows the disjunction of a proposition with its negation to have a non-maximal truth-value, then it looks like the theory allows for the possibility that physical reality is indifferent to the truth of the proposition in question.

However, this argument is flawed on two counts. Firstly, the law of excluded middle alone is not sufficient to motivate classical logic. There are many non-classical substructural logics that satisfy this law. Pertinently in this case, orthodox quantum logic satisfies the law of excluded middle, so this argument could equally well be used to motivate the adoption of non-distributive quantum logic. Secondly, as we will see in a later section, the absence of excluded middle need not entail any kind of indeterminism. In TQT, all physical propositions are assigned definite truth values in a way that violates excluded middle. These truth-values contain concrete physical information and do not require that the propositions to which they are assigned be viewed as 'indeterminate' or non-realist.

Isham and Doering claim that BOOLE 'is compelling in so far as we humans think in a Boolean way'. Of course, this claim is easily countered by the observation that there is no obvious reason that physical reality should be amenable to human thought. However, this perspective does gain some traction when one compares classical logic with orthodox quantum logic. For, non-distributive quantum logic suffers from numerous pathologies that render its status as a logic dubious. For example, quantum logic generally lacks a de- 
duction theorem (see Malinowski [1990]) and does not allow for the definition of a natural implication connective. This renders reasoning with quantum logic highly problematic, and arguably impossible, and not just for humans. Now, it seems reasonable to require that the logic associated with a realist physical theory should at least satisfy certain minimal structural conditions. For example, it should contain some kind of meaningful implication connective satisfying a list of necessary formal properties and be subject to a deduction theorem. Although this is not enough to motivate BOOLE, it is at least a motivation for imposing some kind of formal restriction on the logical structure of realist physical theories.

The third criterion, requiring the existence of 'a space of "microstates" such that specifying a microstate leads to unequivocal truth values for all propositions about the system' will be referred to as 'STATE'. Like PROP, STATE is a highly intuitive condition to impose on realist physical theories. For, the notion of a physical state can be seen as a formalisation of the concept of 'how things are' with respect to the system in question, and it is natural to characterise realist physical theories as telling us 'how things are' with respect to the systems they describe. Indeed, interpreters of quantum theory often ask for an account of 'what the world would be like if quantum mechanics were true'. The fact that this question is problematic is indicative of the failure of quantum theory to satisfy STATE.

\subsection{The spectral presheaf}

4

It is well known that, from a mathematical perspective, KST is a consequence of the existence of non-commuting observables on Hilbert spaces (or, to be more precise, that non-commuting operators are necessary for the application of KST). So, for example, if we let $B(H)$ represent the set of all bounded self-adjoint operators on a Hilbert space $H$ (with $\operatorname{dim}(H) \geq 3$ ), then, because $B(H)$ will generally contain some non-commuting observables, KST will apply, and it will be impossible to simultaneously assign definite values to all of the self-adjoint operators in $B(H)$ in a satisfactory manner. However, if we take any Abelian Von-Neumann subalgebra $V$ of $B(H)$, KST generally will not apply to $V$ since all of the operators in $V$ are pairwise commuting (and the algebra $P(V)$ of projection operators

\footnotetext{
${ }^{4}$ Although we try to keep the use of category theoretic notions to a minimum, some familiarity with the basic notions of category theory and topos theory are assumed throughout the rest of the paper. For a thorough exposition of the relevant notions and a detailed survey of the technical structure of TQT, see (Flori [2013]). Also, for a detailed conceptual introduction to TQT, see (Isham [2011])
} 
in $V$ will be Boolean). So it will, in general, be possible to assign definite values to all of the operators in $V$ without destroying any of the algebraic/functional relationships between them. Intuitively, $V$ corresponds to a kind of 'classical perspective' on the quantum system, in the sense that if we talk only about physical quantities that are represented by operators in $V$, the situation will be analogous to the situation in classical physics, i.e. the algebra of physical propositions will form a Boolean algebra and KST will not apply. This means that it will be possible to define a kind of 'state of the system' from the perspective of $V$, i.e. a two-valued Boolean algebra homomorphism on $P(V)$.

We can give these intuitive notions a rigorous generalisation in the following way: Let $V(H)$ represent the set of all Abelian VonNeumann sub-algebras of $B(H)$. Then, to each $V \in V(H)$, we can associate the set $\Sigma_{V}$ of all homomorphisms from $V$ into $\mathbb{C}$, known as the 'Gelfand spectrum' of $V$. An element of the Gelfand spectrum of $V$ is just a way of assigning a definite value to each of the operators in $V$ in a way that preserves all of the algebraic and functional relations between them. In the terminology established above, the Gelfand spectrum consists of all of the possible states of the system being described, from the perspective of $V$. In TQT, $\Sigma_{V}$ is customarily referred to as the 'local state space' of the system at $V$.

Note that $V(H)$ can be turned into a partially ordered set by the 'is a subalgebra of' relation, denoted by $\subseteq$. Given $V^{\prime} \subseteq V$, we think of $V^{\prime}$ as a 'smaller' classical context than $V$, in the sense that any physical property that can be talked about from the perspective of $V^{\prime}$ can also be talked about from the perspective of $V$. Viewed as a poset, $V(H)$ is often referred to as the 'context category'. We are now ready to define the quantum state space in the formalism of TQT.

Def 2.5.1: The spectral presheaf ${ }^{5} \underline{\Sigma}$ on $V(H)$ is defined by

Objects: Given $V \in V(H)$, the component $\underline{\Sigma}_{V}$ of $\underline{\Sigma}$ at $V$ is the Gelfand spectrum of $V$.

Arrows: Given $i: V^{\prime} \subseteq V, \underline{\Sigma}_{i_{V^{\prime}, V}}: \underline{\Sigma_{V}} \rightarrow \underline{\Sigma_{V^{\prime}}}$

$$
\lambda \mapsto \underline{\Sigma}_{i_{V^{\prime}, V}}(\lambda)=\left.\lambda\right|_{V^{\prime}} 6
$$

Intuitively, $\underline{\Sigma}$ is an assignment that takes each classical perspective $V$ to its associated local state space. Furthermore, if $V^{\prime}$ is a smaller classical perspective than $V$, then $\underline{\Sigma}$ will take the inclusion

\footnotetext{
${ }^{5}$ By a 'presheaf on $V(H)$ ', we will mean a contravariant set-valued functor on $V(H)$.

${ }^{6}$ Where $\left.\lambda\right|_{V^{\prime}}$ represents the restriction of $\lambda$ to $V^{\prime}$.
} 
arrow from $V^{\prime}$ into $V$ and return the function that takes a state $\lambda$ from the local state space of $V$, and returns the state $\left.\lambda\right|_{V^{\prime}}$, which can be thought of as the result of throwing away all the extra physical information that is contained in $V$, but not $V^{\prime}$.

The following theorem is perhaps the most fundamental result of TQT.

Theorem 2.5.2: The Kochen-Specker theorem is equivalent to the fact that the spectral presheaf on $V(H)$ (for $\operatorname{dim}(H) \geq 3$ ) has no global elements ${ }^{7}$

Theorem 2.5.2 shows that, if we interpret the spectral presheaf on $V(H)$ as the state space of a quantum system $S$ described by the Hilbert space $H$, and correspondingly interpret the individual quantum states as the global elements of the spectral presheaf, then KST is actually equivalent to the fact that there are no quantum states for $S$ (the spectral presheaf has no global elements). Later, we will provide a detailed discussion of the notion of the quantum state space in TQT, but for now, it suffices to note that one of the key interpretational moves in TQT is the interpretation of the spectral presheaf as the quantum state space.

We have seen that in TQT, the quantum state space is formalised as a kind of amalgamation of the local, classical state spaces of each of the 'classical perspectives' on $V$ (also referred to as 'contexts'). Thus, we read

'The topos approach emphasises the role of classical perspectives onto a quantum system...One of the main ideas is that all classical perspectives should be taken into account simultaneously' (Döring [2011])

This emphasis on classical perspectives is deeply reminiscent of Bohr's famous 'principle of complementarity'(PC), which is neatly summarised by the claim that

'Talk of the position of an electron has sense only in the context of an experimental arrangement for making a position measurement.' (Bohr, quoted in Gibbins [1987])

The philosophical upshot of PC is that physical propositions about a quantum system can only be made with reference to some fixed classical perspective on that system. This notion is taken seri-

\footnotetext{
${ }^{7}$ Intuitively, a global element of the presheaf $\Sigma$ is the generalisation of the usual set theoretic notion of element to $\underline{\Sigma}$. Formally, a global element of $\underline{\Sigma}$ is an assignment $g: V(H) \rightarrow S E T$ satisfying (i) $g(V) \in \underline{\Sigma}_{V}, \forall V \in V(H)$, and (ii) Given $V, V^{\prime} \in V(H)$, and any arrow $i: V^{\prime} \rightarrow V$, $g$ satisfies $\underline{\Sigma}_{i_{V^{\prime}, V}}(g(V))=g\left(V^{\prime}\right)$.
} 
ously in TQT, and is evident in the way that physical propositions are eventually formalised.

\subsection{The logic of TQT:}

Now that we have access to a suitable notion of a quantum state space, we need to define a corresponding formalisation of physical propositions. Continuing the analogy with the formalism of classical physics, we need to represent physical propositions as a special class of 'subsets' of the quantum state space. But of course, the quantum state space is now formalised as a presheaf, not a set, so we need to generalise this requirement. Specifically, we want to represent physical propositions as a special class of sub-presheaves of $\Sigma$.

Now, in classical physics, the physical proposition $A \in \Delta$ is represented by the subset of the state space that makes the proposition true. This suggests a natural generalisation. Specifically, we represent a physical proposition $A \in \Delta$ in the following way: for each $V \in V(H)$, we take the subset of the local state space at $V$ that makes the physical proposition $A \in \Delta$ true, i.e. we take the set $\left\{\lambda \in \Sigma_{V} \mid \lambda(P)=1\right\}$, where $P$ is the projection operator that corresponds to $A \in \Delta$, in the usual sense. But this is problematic, since it will not generally hold that $P \in V$, and so the local states of $V$ might not be defined on $P$. This problem is the mathematical realisation of PC. A physical proposition will not generally be meaningful for all classical perspectives at once.

So, since we cannot generally be certain that a physical proposition will be meaningful at every classical perspective, we will attempt to 'simulate' the physical proposition in question by approximating to it whenever it is not included in the classical perspective in which we are working. Specifically, we define

Def 2.6.1: The outer daseinisation of a projection operator $P$ for a classical perspective $V \in V(H)$ is defined to be the projection operator given by

$$
\delta^{o}(P)_{V}=\bigwedge\{Q \in P(V) \mid Q \geq P\}
$$

Where $P(V)$ is the set of all projection operators in $V$, and $\geq$ is the usual ordering on the lattice of projections.

Intuitively, the outer daseinisation of $P$ at $V$ is $V$ 's best approximation to $P$ 'from above'. Of course, if $P \in P(V)$, then this approximation will just be $P$ itself. If $P \notin V$, then this approximation will be the strongest proposition that can be asserted from the classical perspective represented by $V$ that is implied by $P$. The idea is that in this case, $V$ does not contain enough information about the 
system to allow us to assert $P$, but the outer daseinisation of $P$ at $V$ is the closest thing to $P$ that we are allowed to say, given only the information contained in $V$. It may be that $P$ is just a proposition $A \in \Delta$, where $A$ is the momentum of the system. Now, if $V$ contains the projection $Q$ corresponding to $B \in \Theta$, where $B$ represents the position of the system, and $\Theta$ is a sufficiently small interval, then $\mathrm{P}$ and $\mathrm{Q}$ won't commute (by the uncertainty principle) and so we will have $P \notin V$. In this case, $\delta^{o}(P)_{V}$ might be the projection corresponding to the proposition $A \in \Gamma$, where $\Delta \subseteq \Gamma$ and $\Gamma$ is a large enough interval to allow us to assert $A \in \Gamma$ at $V$ without violating the uncertainty principle.

Returning to the analogy with classical physics, we think of each such approximation to a physical proposition $A \in \Delta$ at a classical perspective $V$ as the 'local proposition' to which $A \in \Delta$ corresponds at $V$, and we represent each such local proposition with the subset of the corresponding local state space that makes it true.

Def 2.6.2: Let $V \in V(H), P \in P(H)$ (where $P(H)$ is the lattice of all projection operators on $H$ ). Then define

$$
S_{\delta^{o}(P)_{V}}=\left\{\lambda \in \underline{\Sigma}_{V} \mid \lambda\left(\delta^{o}(P)_{V}\right)=1\right\}
$$

So $S_{\delta^{\circ}(P)_{V}}$ is the set of the possible states of $V$ that make $V$ 's approximation to $P$ true.

We are now ready to represent any quantum proposition as a sub-presheaf of the spectral presheaf.

Def 2.6.3: Let $P \in P(H)$. Then we define $P$ 's outer daseinisation presheaf $\delta^{o}(P)$ on $V(H)$ by

Objects: Given $V \in V(H)$, the component $\underline{\delta^{o}(P)}{ }_{V}$ of $\underline{\delta^{o}(P)}$ at $V$ is given by $\frac{\delta^{o}(P)_{V}}{=}=S_{\delta^{o}(P)_{V}}$

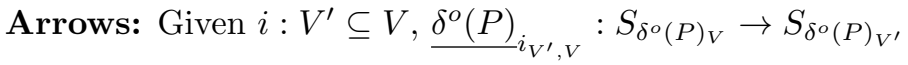

$$
\left.\lambda \mapsto \lambda\right|_{V^{\prime}}
$$

Note that this is well defined since if $\lambda \in S_{\delta^{o}(P)_{V}}$, then $\lambda\left(\delta^{o}(P)_{V}\right)=$ 1. Now, since $V^{\prime} \subseteq V$, we have

$$
\delta^{o}(P)_{V}=\bigwedge\{Q \in P(V) \mid Q \geq P\} \leq \bigwedge\left\{Q \in P\left(V^{\prime}\right) \mid Q \geq P\right\}=
$$
$\delta^{o}(P)_{V^{\prime}}$. So $\delta^{o}(P)_{V} \leq \delta^{o}(P)_{V^{\prime}}$. 
So, since $\lambda$ is a homomorphism and $\lambda\left(\delta^{o}(P)_{V}\right)=1$, we have that $\lambda\left(\delta^{o}(P)_{V^{\prime}}\right)=1$, proving that the presheaf is well defined.

Note that we refer to the general fact that $\delta^{o}(P)_{V} \leq \delta^{o}(P)_{V^{\prime}}$ for $V^{\prime} \subseteq V$ as 'coarse graining'.

Thus, in analogy with classical physics, we represent a proposition as a collection of subsets of local state spaces, one for each $V \in V(H)$. Specifically, for $V \in V(H)$, we choose the subset of $V$ 's local state space that makes $V$ 's approximation to the proposition true. The following definition will be useful,

Def 2.6.4: A sub-object (sub-presheaf) $\underline{S}$ of the spectral presheaf $\underline{\Sigma}$ is a presheaf on $V(H)$ such that (i) $\forall V \in V(H)\left(\underline{S}_{V} \subseteq \underline{\Sigma}_{V}\right)$ (ii) Given $i: V^{\prime} \subseteq V, \Sigma_{i_{V^{\prime}, V}}\left(\underline{S}_{V}\right) \subseteq \underline{S}_{V^{\prime}}$

Now, it should be noted that for any $P \in P(H)$ and for any $V \in V(H)$, the set $S_{\delta^{\circ}(P)_{V}} \subseteq \underline{\Sigma}_{V}$ is always a clopen subset of $V$ 's Gelfand spectrum $\underline{\Sigma}_{V}$ (where $\underline{\Sigma}_{V}$ is given the weak * topology). Together with 2.6.4, this fact motivates the following definition,

Def 2.6.5: A clopen sub-object $\underline{S}$ of the spectral presheaf is a subobject $\underline{S}$ of $\underline{\Sigma}$ such that $\forall V \in V(\bar{H}) \underline{S}_{V}$ is a clopen subset of $\underline{\Sigma}_{V}$

So, in TQT, we represent propositions as clopen sub-objects of the spectral presheaf. Note that for each $V \in V(H)$, the set $c l\left(\underline{\Sigma}_{V}\right)$ of clopen subsets of $V$ 's Gelfand spectrum is in bijective correspondence with the set $P(V)$ of all projection operators in $V$. Thus, any clopen subobject $\underline{S}$ of the spectral presheaf defines a 'local proposition' $P_{\underline{S}_{V}} \in P(V)$ for each classical perspective $V$. It is easily seen that coarse graining will apply to these local propositions. For, if $V^{\prime} \subseteq V$ then $\Sigma_{i_{V^{\prime}, V}}\left(\underline{S}_{V}\right) \subseteq \underline{S}_{V^{\prime}}$. So, given $\lambda \in \underline{\Sigma}_{V}$ such that $\lambda\left(P_{S_{V}}\right)=1$, we have that $\lambda \in \underline{S}_{V}$ and hence $\left.\lambda\right|_{V^{\prime}} \in \underline{S}_{V^{\prime}}$, which implies $\left.\lambda\right|_{V^{\prime}}\left(P_{\underline{S}_{V^{\prime}}}\right)=1$, i.e. $\lambda\left(P_{\underline{S}_{V^{\prime}}}\right)=1$. This proves that $P_{\underline{S}_{V}} \leq P_{\underline{S}_{V^{\prime}}}$.

So we can think of $\underline{S}$ as a kind of 'global proposition' whose local components get more general as we lose information by moving to smaller classical perspectives. Note that it will not generally hold that $\underline{S}=\delta^{o}(P)$ for some $P \in P(H)$. The outer daseinisation presheaves are only a special subclass of the clopen sub-objects of the spectral presheaf. By interpreting the set $S u b_{c l}(\underline{\Sigma})$ of all clopen sub-objects of the spectral presheaf as the set of all physical propositions that can be made about the quantum system, we are making a significant interpretational leap in order to obtain a logical structure that is strictly richer than that of traditional quantum logic. We consider the philosophical merits of this interpretational move 
in detail in section 4 .

We have now defined the algebra of propositions for TQT, and are in a position to examine whether it satisfies Isham and Döring's stringent condition for realist physical theories, BOOLE. The following result is important in this respect:

Theorem 2.6.6: Define the ordering relation $\leq$ on $S u b_{c l}(\underline{\Sigma})$ as follows: Given $\underline{S}, \underline{T} \in S u b_{c l}(\underline{\Sigma}): \underline{S} \leq \underline{T} \leftrightarrow\left(\forall V \in V(H): \underline{S}_{V} \subseteq \underline{T}_{V}\right)$. Then $\leq$ turns $S u b_{c l}(\underline{\Sigma})$ into a complete, bounded distributive lattice. Specifically, $S u b_{c l}(\underline{\Sigma})$ is a complete Heyting algebra.

We will consider the philosophical significance of 2.6.6 in detail later in the paper. For now, there is one more salient aspect of the formalism of TQT that we need to be acquainted with.

Recall that in classical physics, it was always possible to assign classical truth values to all of the physical propositions associated with a classical system in an unproblematic way. Specifically, this was achieved by assigning a state to the system in question. Similarly, KST tells us that it is not possible, in the Hilbert space formalism, to assign truth values unproblematically to all of the physical propositions associated with a quantum system, it is generally possible, given a state vector $|\psi\rangle$, to assign truth values unproblematically to some physical propositions. Specifically, any physical proposition $A \in \Delta$ that is represented by a projection operator $P$ that has $|\psi\rangle$ as one of its eigenvectors will be assigned a classical truth value by $|\psi\rangle$. Now, in TQT, we do not yet have access to any notion of a quantum state that can assign truth values to physical propositions (i.e. to elements of $\left.S u b_{c l}(\underline{\Sigma})\right)$. But this is a problematic state of affairs. For, surely, a basic condition that any meaningful physical theory should fulfill is that it should have some mechanism for assigning truth values to at least some of the physical propositions with which it is concerned. Indeed, this requirement is implied by the first criterion of realism that was posited by Isham and Döring, PROP. So, in order to satisfy its own philosophical motivations, TQT needs to supply a procedure for assigning truth-values to physical propositions.

At this stage, the topos-theoretic structure of the formalism of TQT becomes indispensable. In TQT, we represent both the quantum state space and physical propositions as presheaves over the context category. Generalising, we can define the category $S E T^{V(H)^{o p}}$ of all presheaves over the context category. ${ }^{8}$ Now, it is well known that $S E T^{V(H)^{o p}}$, as a presheaf category, is actually a topos. The technical details of this fact are mainly unimportant for the purposes of this article. Intuitively, what this means is that $S E T^{V(H)^{o p}}$

\footnotetext{
${ }^{8}$ Morphisms in this category are just presheaf morphisms, in the standard sense. The explicit definition will be unimportant in what follows
} 
can be thought of as a kind of mathematical universe that models all intuitionistically valid mathematics. The elements of this universe can be thought of as sets that 'vary' over the context category, i.e. for each element $V$ of the context category, they give you some (classical, non-varying) set. In this sense, we can interpret the standard model of classical set theory as a presheaf topos. Specifically, the classical set theoretic hierarchy can be reformalised as the topos of presheaves over a trivial one-element poset $P$. So any set $x$ is uniquely associated with the presheaf that takes the only element $p \in P$ to the set $x$. Thus, $S E T^{V(H)^{o p}}$ can be seen as a natural generalisation of the set theoretic hierarchy, i.e. one in which the sets are allowed to 'vary' over the context category. The main thing that is lost in this generalisation is that we are no longer working in a model of the whole of classical mathematics. Rather, we are working in a model of all of intuitionistic mathematics, and the logic that holds in $S E T^{V(H)^{o p}}$ is intuitionistic rather than classical.

The relevance of all this to our current problem, i.e. defining a procedure for assigning truth values to physical propositions in TQT, is that $S E T^{V(H)^{O P}}$ has a very rich internal structure that allows us to simulate much of the structure of the set-theoretic hierarchy. In particular, like the set-theoretic hierarchy, $S E T^{V(H)^{o p}}$ has its own algebra of truth values. Recall that we can reformulate the settheoretic hierarchy as the topos of presheaves $S E T^{P^{o p}}$ over a trivial one-element poset $P$. Now, in classical set theory, the truth value algebra is just the two element Boolean algebra $\{0,1\}$. By means of the following definition, we can find the natural generalisation of $\{0,1\}$ to $S E T^{V(H)^{o p}}$.

Def 2.6.7: Let $P$ be a poset, and let $p \in P$. A sieve on $p$ is a subset $S \subseteq P$ such that (i) $\forall q \in S(q \leq p)$, (ii) If $q \in S$ and $r \in P, r \leq q$, then $r \in S$, i.e. $S$ is a collection of elements of $P$ 'below' $p$ that is closed downwards under the ordering on $P$. We call the maximal sieve on $\mathrm{p},\{q \mid q \leq p\}$, the 'principal sieve on p', denoted $\downarrow p$.

Now, if $P$ is the trivial one-element poset with a sole element $p \in P$, then there are exactly two sieves on $p$, i.e. the empty sieve and the maximal sieve $S=\{p\}$. We know that the set-theoretic universe is just the presheaf topos $S E T^{P^{o p}}$, and that the truth value algebra for this universe is just $\{0,1\}$, where 0,1 are some arbitrary sets. So, since there are only two sieves on $p \in P$, we take 0 and 1 to be the empty and maximal sieves on $p$, respectively. Thus, the classical truth values of set theory can be identified with the sieves on the sole element of the trivial one-element poset.

For our current purposes, the most salient consequence of the fact that $S E T^{V(H)^{o p}}$ is a topos is that there exists a distinguished object (presheaf) in $S E T^{V(H)^{o p}}$, called 'the subobject classifier' and 
denoted $\Omega$, whose global elements are interpreted as the truth-values of the mathematical universe $S E T^{V(H)^{o p}}$. Intuitively, $\Omega$ is the translation of the two-element Boolean algebra in $S E T^{V(\bar{H})^{o p}}$. The following standard result gives an explicit description of $\underline{\Omega}$.

Proposition 2.6.8: The subobject classifier in $S E T^{V(H)^{o p}}$ is the presheaf, $\underline{\Omega}$, defined by

Objects: Given a Context $V$, the set $\underline{\Omega}_{V}$ is defined to be the set of all sieves on $V$.

Arrows: Given a morphism $i_{V, V^{\prime}}: V \rightarrow V^{\prime}$ in $V(H)$, the associated function $\underline{\Omega}_{V, V^{\prime}}: \underline{\Omega}_{V^{\prime}} \rightarrow \underline{\Omega}_{V}$ is defined to take a sieve $S$ on $V^{\prime}$ to $\underline{\Omega}_{V, V^{\prime}}(S)=\left\{V^{\prime \prime} \in \overline{S \mid V^{\prime \prime}} \subseteq \bar{V}\right\}$.

Proposition 2.6.8 tells us that the subobject classifier in $S E T^{V(H)^{o p}}$ is a direct generalisation of the two-element Boolean algebra. For, just as the two-element Boolean algebra can be interpreted as an assignment that takes each element of the one-element poset to the set of all sieves on that element, the subobject classifier is just the presheaf that takes each classical context $V \in V(H)$ and returns the set of all sieves on that context. Furthermore, just as a classical truth value can be interpreted as a sieve on the sole element of the one-element poset, a truth value in $S E T^{V(H)^{o p}}$ is really just a way of picking, for each $V \in V(H)$, one sieve on $V$.

Now, one of the fundamental ideas behind TQT is that $S E T^{V(H)^{o p}}$ is somehow the natural mathematical habitat for a formalisation of quantum theory. Working on the basis of this assumption, it is natural to conclude that when assigning truth values to physical propositions in TQT, we should use the truth-values of $S E T^{V(H)^{o p}}$, not the classical truth-values of set theory. Thus, our problem has taken a new form. We are no longer attempting to assign classical truth-values to physical propositions. Rather, we are trying to assign truth values 'inside' of $S E T^{V(H)^{o p}}$.

In the Hilbert space formalisation of quantum theory, any statevector $|\psi\rangle$ automatically assigned classical truth values to all of the physical propositions represented by projection operators of which $|\psi\rangle$ is an eigenvector. Similarly, in order to assign truth-values to physical propositions in TQT, we begin by defining use state-vectors from the relevant Hilbert space.

Def 2.6.9: The truth value of a physical proposition, represented in TQT by an element $\underline{S}$ of $S u b_{c l}(\underline{\Sigma})$, relative to a pure state $|\psi\rangle$, is defined context-wise: given a context, $V \in V(H)$, we define the truth value of $\underline{S}$ relative to $|\psi\rangle$ to be $\left\|\underline{S} \in T^{\psi}\right\|_{V}=$

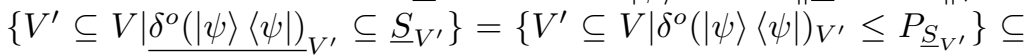


$\left\{V^{\prime} \subseteq V \mid\left\langle\psi\left|P_{\underline{S}_{V^{\prime}}}\right| \psi\right\rangle=1\right\}$

The truth value assigned to $\underline{S}$ at a context $V$ by the pure-state $|\psi\rangle$ is the collection of all sub-contexts of $V$ whose corresponding 'local proposition' $P_{\underline{S}_{V^{\prime}}}$ is implied by that context's approximation to the proposition that is normally interpreted as 'the state of the system is $|\psi\rangle^{\prime}$. The crucial point to note at this stage is that these truth value assignments are in bijective correspondence with the one dimensional subspaces of the original Hilbert space $H$. Intuitively, the idea is that the truth value of a clopen subobject $\underline{S}$ with respect to a state vector $|\psi\rangle$ tells you at which classical perspectives the proposition 'the state of the system is $|\psi\rangle$ ' implies the proposition represented by $\underline{S}$. At first blush, the physical significance of these truth values seems obscure. In section 4 , we will give a detailed philosophical analysis of their meaning, and attempt to provide a natural physical interpretation.

\section{Interpreting States in TQT}

We have seen that TQT is motivated largely by KST and the way it excludes the possibility of the Hilbert space formalisation of quantum theory ever satisfying Isham and Döring's third criterion for realist physical theories (the existence of a space of microstates whose elements assign truth values to all of the relevant physical propositions in a consistent manner), STATE. We are now in a position to assess whether or not TQT fares any better than the orthodox Hilbert space formalism with respect to satisfying STATE.

In the literature, the spectral presheaf of a quantum system is often described as 'the analogue of the state space of a classical system' (Döring [2010]). This idea can really be thought of as the fundamental interpretational conceit of TQT, as much of the overall interpretation of the formalism is justified by the conception of the spectral presheaf as the quantum state space. This all leads to the following natural question: if the spectral presheaf plays the role of the state space in TQT, then what plays the role of the individual quantum states?

The first, and most intuitive, possible response to this question is to claim that the global elements of the spectral presheaf represent the individual quantum states. For, what is a state space other than an object whose elements are states? However, we have already seen that the non-existence of global elements of the spectral prehseaf (for dimension greater than 2) is equivalent to KST. So, if we decide to take this line and interpret the individual states of the system as global elements of the spectral presheaf, then we will be forced to conclude that, in general, there are no individual quantum states, 
and that the quantum state space is, in this specific sense, empty.

At this point, one might be inclined to discount this interpretational move as implausible. For, surely, whatever formal objects we use to represent quantum states, we should always at least be sure of their existence. However, there is a possible response to this kind of objection. Specifically, recall that in classical physics, states can be identified with homomorphisms between the lattice of physical propositions and the lattice of truth values. If, taking analogy with classical physics as our guide, we characterise quantum states in the same way, then KST can be interpreted as saying that there are no quantum states. Then, given the interpretation of global elements of the spectral presheaf as individual quantum states, the fact that KST is equivalent to the non-existence of these global elements appears very natural.

Let us suppose for the moment that this response is adequate. We are still faced with the question of whether or not this interpretational move satisfies STATE. In particular, we want to know whether a global element of the spectral presheaf will generally assign truth-values to physical propositions in an unproblematic way. But since, in general, there are no such global elements, this condition is trivially satisfied.

Philosophically, this looks uninformative. Although, technically, this representation of individual quantum states does satisfy STATE, the victory is a Pyhrric one. For, the notion of Realism with which we have been working has been defined primarily in terms of the possibility of assigning truth values to physical propositions. So, we should surely require that our theory should contain some mechanism for assigning truth values to physical propositions (indeed, this requirement is really just Isham and Döring's first criterion, PROP). But any such mechanism will make no mention of global elements of the spectral presheaf, since there are none. Following our guiding principle of analogy with classical physics, surely we should interpret these truth-value assignments as the individual states of the system, contradicting the interpretation of the global elements of the spectral presheaf as the individual quantum states. The very principle that justified this interpretational manoeuvre (analogy with classical physics) also rules it out.

In fact, in the previous section, we saw the mechanism that is generally used to assign truth values to physical propositions in TQT. This provides us with another candidate for the formal representation of individual quantum states, i.e. the truth value assignments. Again, this kind of move is justified by analogy with classical physics, where states and truth value assignments can be unproblematically identified.

The mechanism in question uses pure state vectors in the original Hilbert space $H$ to assign truth values to physical propositions. 
Indeed, truth-value assignments in TQT are in bijective correspondence with pure-state vectors in $H$. So, under this proposal, the individual quantum states of TQT correspond to one-dimensional subspaces of $H$.

This is essentially the same as the interpretation that is prevalent in the literature (see e.g Döring [2010]). To be precise, the usual proposal is that we represent individual quantum states by the daseinisation presheaves corresponding to projections onto onedimensional subspaces of the original Hilbert space $H$. So, given some $|\psi\rangle \in H$, we interpret the object $\delta^{o}(|\psi\rangle\langle\psi|)$ as a possible state of the system, and call it a 'pseudo-state'. Since the daseinisation operation is injective, this is equivalent to interpreting the one dimensional subspaces of the original Hilbert space as the possible states of the system.

Although this is the dominant approach to representing individual states in TQT, it is immediately open to several possible criticisms. Firstly, if the individual states of the quantum system are really just the one-dimensional subspaces of $H$, then what reason do we have to interpret the spectral presheaf as the quantum state space? For, the one-dimensional subspaces of $H$ do not correspond to elements of the spectral presheaf (since there are none). Indeed, there is no obvious relationship between the one-dimensional subspaces of $H$ and the spectral presheaf that independently justifies the interpretation of the former as the individual states of the quantum system and the latter as the state space in which they live.

This leads to a further, and much graver criticism. If, in TQT, the individual states are represented by one-dimensional subspaces of $H$, then surely $H$ is the most natural candidate for representing the quantum state space. But then, we're right back where we started, in orthodox Hilbert space quantum theory, and the whole enterprise collapses.

The advocate of TQT might respond that they are only attempting to provide a reformulation of quantum theory, and so it should be expected that the quantum states of TQT can be bijectively mapped back to the one-dimensional subspaces of the relevant Hilbert space. But this seems unsatisfactory. For, if we want our reformulation to exactly preserve the quantum states of the original Hilbert space formalism, then surely we should also want it to preserve the relationship between those states and the state space to which they correspond. As we have seen, this relationship is not preserved. In the Hilbert space formalism, quantum states are elements of the state space, in TQT they are not. This raises the question 'why should we interpret the spectral presheaf as the quantum state space?'.

Furthermore, there is a definite sense in which TQT is more than just a straightforward reformulation of Hilbert space quantum theory. Consider, for example, the physical propositions of TQT. As we 
will discuss in section 5 , there are strictly more physical propositions in TQT than there are in the Hilbert space formalism. In particular, TQT posits the existence of physical propositions that cannot be represented by projection operators. This is a clear example of a way in which TQT does more than simply provide a new face for the Hilbert space formalism. It introduces fundamentally new structure that cannot be reduced to the familiar structure of the old formalism. What's more, this new structure is used to license bold interpretational claims by TQT's proponents. Returning to the example, TQT's extra new physical propositions are necessary for obtaining the intuitionistic logical structure of the formalism, which is central to the project's philosophical vision.

In order to avoid these criticisms, one could decide to weaken the condition STATE and require only that states correspond to probability measures on the clopen subobjects, rather than requiring that they correspond to truth-value assignments. Philosophically, this looks like a strange move for the proponent of TQT to make, since states in the original Hilbert space formalism also satisfy this weakened form of STATE (i.e., they induce probability measures on the lattice of projections), which means that a large part of the motivation is lost. However, let us suppose that the advocate of TQT has an answer to this kind of worry, and is willing to argue for a weaker form of realism. Then, they might be inclined to propose that individual states in TQT should be represented by probability measures on the lattice of clopen subobjects.

Since we are in a topos-theoretic setting, and the advocate of TQT wants everything to be defined in the topos of presheaves over $V(H)$, we want our probability measures to take values in an object that lives in this topos (just as we wanted to use truth-values that live in this topos). Towards this end, it is possible to define an analogue of the closed unit interval in $S E T^{V(H)^{o p}}$ as follows,

Def 3.1: The unit interval object in $S E T^{V(H)^{o p}}$ is the presheaf, $[0,1] \leq$, defined by

Objects: Given a Context $V$, the set $[0,1] \leq_{V}$ is defined to be

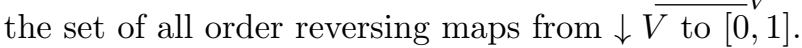

Arrows: Given an inclusion morphism $i_{V, V^{\prime}}: V^{\prime} \rightarrow V$ in $V(H)$,

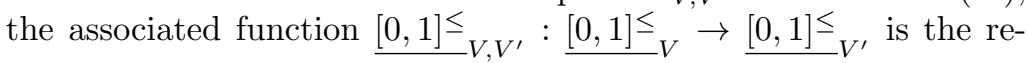
striction map that given $f \in \underline{[0,1] \leq}{ }_{V}$, returns the restriction $\left.f\right|_{\downarrow V^{\prime}}$ of $f$ to $\downarrow V^{\prime}$.

For current purposes, the technical details of this definition are unimportant (see Döring and Isham [2012] for a full technical explanation). What matters is that this object plays the role of the closed 
unit interval in $S E T^{V(H)^{o p}}$. Accordingly, the notion of a probability measure on the lattice of clopen subobjects of the spectral presheaf is defined as taking values in $[0,1] \leq$, not $[0,1]$. Generally, a probability measure is defined to be a presheaf morphism from $S u b_{c l}(\underline{\Sigma})$ to $[0,1] \leq$ satisfying the category-theoretic translations of the usual probability axioms (for details see Isham and Döring [2012]). The suggestion then is that these presheaf morphisms play the role of individual quantum states in TQT.

However, it turns out that this approach leads directly to the same problem that occurred when we tried to interpret truth-value assignments on $S u b_{c l}(\underline{\Sigma})$ as quantum states. For, in (Isham and Döring [2012]), it was shown, using Gleason's theorem, that probability measures on $S u b_{c l}(\underline{\Sigma})$ are in bijective correspondence with density matrices on the original Hilbert space $H$. In particular, any such probability measure corresponds to the trace measure of some density matrix $\rho$. So, the proposal that the quantum states of TQT should be represented by probability measures is really equivalent to the proposal that quantum states should be represented by density matrices. But then, we arrive back again at the Hilbert space formalism, where mixed states are represented by density matrices. Again, given this interpretation, it seems natural to claim that the original Hilbert space $H$ is really playing the role of the quantum state space, not the spectral presheaf. Certainly, it seems that given this interpretation, $H$ is at least as good a candidate for the title of 'quantum state space' as the spectral presheaf. For, as we have seen, density matrices, probability measures on the clopen subobjects of the spectral presheaf, and probability measures on the lattice of projection operators are all really the same thing.

At any rate, one might be inclined to argue, even if the proponent of this interpretation does provide an answer to this objection, they are only providing a notion of quantum state that satisfies the weakened version of STATE, where states correspond to probability measures rather than truth value assignments. They have still not shown that TQT satisfies the realist criteria that have been set out. However, it turns out that this kind of objection is easily countered. For, in TQT, mixed states can also be used to define truth value assignments that generalize the truth value assignments that correspond to pure states in a natural way. Indeed, in (Isham and Döring $[2012])$, it was shown that any probability measure on $S u b_{c l}(\underline{\Sigma})$ can be replaced, without loss of information, by a truth value assignment defined by the mixed state corresponding to that measure. Although we do not have the room to explore this impressive result in detail here, it is worth noting that it hs been used to justify a form of realism about quantum probabilities in TQT. Since, the argument goes, probability measures on $S u b_{c l}(\underline{\Sigma})$ correspond to mixed states which correspond (uniquely) to truth value assignments, we can think of 
probability measures on $S u b_{c l}(\Sigma)$ as truth value assignments. And, furthermore, since we are realists about truth values (i.e. we think that they represent genuine, real properties of the system that exist at all times), we can be realists about probability measures, and treat them as genuine properties of the system. As Isham and Döring put it,

'this approach to probability theory allows for a new type of non-instrumentalist interpretation that might be particularly appropriate in propensity schemes' (Isham and Döring [2012])

Unfortunately, we cannot provide a detailed analysis of this kind of claim here. The key fact to bear in mind is that although, at first blush, it looks like probability measures on $S u b_{c l}(\underline{\Sigma})$ will be too weak to provide a realist notion of quantum states, it turns out that they do actually correspond to truth value assignments, and so do satisfy STATE. One interesting consequence of this is that TQT does not appear to draw any kind of fundamental ontological distinction between pure state vectors in the original Hilbert space and density matrices acting on that Hilbert space. Both allow us to define truth value assignments on $S u b_{c l}(\underline{\Sigma})$, and so provide notions of quantum state that satisfy the key criterion STATE.

In summary, we have considered three possible candidates for the representation of individual quantum states in TQT. Firstly, we saw that interpreting global elements of the spectral presheaf as quantum states is problematic because of KST, which tells us that they do not generally exist, and so can play no role in the assignment of probabilities or truth-values to physical propositions. Secondly, we saw that the common convention of interpreting the daseinisations of projections onto one-dimensional subspaces of the original Hilbert space is also problematic because it reduces to interpreting pure state vectors in the original Hilbert space as quantum states, which suggests that the original Hilbert space, not the spectral presheaf, should be interpreted as the quantum state space. Finally, we saw that interpreting probability measures on $S u b_{c l}(\underline{\Sigma})$ as quantum states is equally problematic because it reduces to interpreting density matrices as quantum states, which again suggest that the original Hilbert space is really playing the role of the quantum state space. Generally, the problem is that TQT proposes a new formal representation of the quantum state space, without providing a new formal representation of individual quantum states. Conceptually, this seems problematic because it seems to imply that the new representation of the quantum state space is not actually capable of carrying out the work done by its predecessor. 


\section{Interpreting Truth Values and Clopen Subobjects in TQT}

\subsection{Interpreting the truth values}

In section 2, we saw that physical propositions in TQT are assigned truth values 'inside' of $S E T^{V(H)^{o p}}$. These truth values are a natural generalisation of the classical truth values familiar from set theory, obtained by extending the poset over which we define our presheaves. However, we have not yet discussed the physical meaning of these truth values, i.e. what does it mean to assign a physical proposition a truth value within $S E T^{V(H)^{o p}}$ ? In this section, we will examine the physical significance of these truth values and attempt to provide them with a natural physical interpretation.

The first fact to note is that for any context $V$, the set $\underline{\Omega}_{V}$ of all sieves on $V$ is a Heyting algebra under some appropriately chosen set theoretic operations, with maximal and minimal elements $\downarrow V$ and $\emptyset$, respectively. Recall that this is a direct generalization of classical set theory, where $V$ will be the only element of a trivial poset and $\underline{\Omega}_{V}$ will be the two-element Boolean algebra. So we can think of local truth values as belonging to an algebraic structure that generalizes the familiar two element Boolean algebra of classical set theory.

It turns out that we can also obtain a Heyting algebraic structure when we consider 'global truth values' (global elements of $\underline{\Omega}$ ). For, it is a basic fact of topos theory that the collection, $\Gamma(\underline{\Omega})$, of all global elements of the subobject classifier in a topos will always form a Heyting algebra. So, we obtain Heyting algebras of truth values at both the local and the global levels. So on a mathematical level, these truth values appear unproblematic. They inhabit algebraic structures that are familiar from intuitionistic logic, and can be seen as direct generalisations of classical truth value algebras.

The fact that these truth value algebras generalize the classical two-element Boolean algebra can also be seen to have physical significance. In classical physics, if we consider the collection of all physical quantities associated with a system (corresponding to $B(H)$ ), we will not find any incompatible observables, and so we will not have to move to the category of commutative sub-algebras of observables. We only need one context, i.e., the collection of all relevant physical quantities. So our poset will be trivial, and we will only obtain classical truth values. So there is a definite sense in which the generalisation of classical truth values in TQT is necessitated by the existence of incompatible observables. If there were no incompatible observables, we would only need one context, and every proposition would obtain a classical truth value. In this sense, TQT represents a mathematical generalisation of classical set theory 
and a physical generalisation of classical physics.

Recall the explicit form of the truth value assignments in TQT, i.e. given a clopen subobject $\underline{S}$ and a pure state vector $|\psi\rangle$, the truth value of $\underline{S}$ relative to $|\psi\rangle$ at a context $V$ is $\left\{V^{\prime} \subseteq V \mid \delta^{o}(|\psi\rangle\langle\psi|)_{V^{\prime}} \leq\right.$ $\left.P_{\underline{S}_{V^{\prime}}}\right\}$. For now, let us focus on the case in which $\underline{S}$ is $\delta^{o}(P)$ for some projection $P$. In this case, $P_{S_{V}}=\delta^{o}(P)_{V}$. So, what the truth value is telling you here is at which classical perspectives the approximation to the proposition 'the state of the system is $|\psi\rangle$ ' implies the corresponding approximation to the proposition $P$. Whenever this holds, the expectation value of the approximation of $P$ with respect to $|\psi\rangle$ will always be 1 .

In orthodox Hilbert space quantum theory, a proposition having expectation value 1 at a state is interpreted as meaning that the proposition is completely true at that state. So the truth value of a proposition $\delta^{\circ}(P)$ relative to a state-vector $|\psi\rangle$ at a context $V$ tells you about those sub-contexts of $V$ whose approximation to $P$ is completely true at $|\psi\rangle$, in the sense of orthodox Hilbert space quantum theory. Intuitively, what this kind of truth value tells you is how far you have to generalize $V$ 's approximation to $P$ before it becomes true with respect to $|\psi\rangle$. Clearly, then, these truth values encode a great deal of physically significant information.

Compare this with, for example, Reichenbach's proposal of a third 'indeterminate' truth value for problematic quantum propositions:

'The starting point of Reichenbach's considerations was Heisenberg's principle of indeterminacy, which states that a microphysical quantity has no definite value if a complementary quantity has already been measured. Heisenberg and other physicists considered statements about these unmeasurable quantities as "meaningless". For Reichenbach, however, this term was unacceptable, since as a logical empiricist he subscribed to the ideal of a scientific language not containing any meaningless statements at all...To solve this difficulty, Reichenbach now proposed to reserve the label "meaningless" to propositions about quantities unmeasurable in any physical situation, and to call those propositions which might have been true or false under different circumstances "indeterminate" , (Kamlah [1981]).

The philosophical advantages of the truth values used in TQT over those offered by an approach like Reichenbach's are manifold: Firstly, Reichenbach's truth values are very uninformative compared to the sieve valuations outlined above. All propositions about quantities that are incompatible with observables about which we already have information are assigned the same truth value: 'indeterminate'. In contrast, in TQT, these kinds of propositions will generally be assigned varying truth values, all of which encode a great deal of 
information about the ways in which the proposition can be approximated at different contexts, and how these approximations behave. Reichenbach's third truth value is completely blind to the differences between propositions of this sort, and seems to function as nothing more than a generic label for problematic quantum propositions. Simply labeling these propositions as 'indeterminate' does not teach us anything interesting, and it only serves to mystify these propositions further. In contrast, the sieve truth values of TQT have a clear meaning. We know exactly what it is that they are telling us, i.e. whether it is possible to generalise (by moving to a smaller context) an approximation to a quantum proposition in a way that makes the generalisation true, and if so, how.

Secondly, the truth values of TQT are not arbitrarily selected. They are a fundamental part of the mathematical framework of this formalisation of quantum mechanics. The truth values in a topos are always uniquely determined. As Butterfield puts it,

'One common reason for being suspicious of notions of partial truth, and of many valued logics, is the idea that the proposed notions are arbitrary: that other definitions, e.g. about how to define truth functional connectives, would be just as well motivated by the logico-semantic phenomena or intuitions appealed to, as are the proposed definitions. But this reason does not apply here. For ... in any topos, the collection of generalized truth-values (the sub-object classifier $\Omega$ ) is completely fixed by the structure of the topos; (and in general, there is no other topos with a similar but different $\Omega$ that one can argue to be as well motivated as the given one). In short, the many valued logics that arise in a topos are not arbitrary' (Butterfield [1999]).

Finally, we have seen that the non-classical partial truth values of TQT arise as a natural generalization of the truth values in classical physics and set theory. Indeed, the truth values of TQT are determined by the mathematical structure of the context category, and hence of the Hilbert space associated with the quantum system. Different Hilbert spaces will give rise to different context categories and different truth values. So the truth values of TQT bear a close relationship to the mathematical structure of the quantum formalism. Again, Reichenbach's 'indeterminate' truth value appears arbitrary and uninformative in contrast. It bears no relationship to the formalisation of the properties of a quantum system, and does not arise as a natural generalization of the truth values of classical physics.

Thus, we have seen that the truth values employed by TQT are well motivated and intuitive from both a mathematical and a physical point of view. 


\subsection{Interpreting $S u b_{c l}(\underline{\Sigma})$}

In section 2, we saw that TQT represents physical propositions by clopen subobjects of the spectral presheaf, so that $S u b_{c l}(\underline{\Sigma})$ represents the lattice of physical propositions, analogous to the lattice of measurable subsets of the phase space in classical physics, or the lattice of projection operators in orthodox Hilbert space quantum theory. One of the fundamental results of TQT is that this lattice is actually a Heyting algebra under some very natural operations, and so the logic that corresponds naturally to the theory is intuitionistic. Recall that one of Isham and Döring's realist criteria, BOOLE, was that the logic of our theory should be Boolean. So TQT fails to satisfy BOOLE. However, the fact that the lattice of physical propositions is now a distributive Heyting algebra rather than a non-distributive orthomodular lattice is commonly portrayed as a major advantage of TQT. The idea is that intuitionistic logic is, in some intuitive sense, 'closer' to classical logic than traditional quantum logic is.

This kind of claim is difficult to evaluate, since there is no universally accepted metric for measuring the proximity of one logic to another. Although intuitionistic negation allows us to recover distributivity and all the corresponding structural properties that are absent from orthomodular quantum logic, it forces us to surrender double negation elimination and the law of excluded middle, which are both still present in orthomodular quantum logic, where the negation operation behaves classically.

Certainly, though, quantum logic suffers from many well publicised formal pathologies (like the absence of a deduction theorem (see Malinowski [1990]) or a canonical implication operation), and it is probably fair to say that it is technically not as well understood as intuitionistic logic. It could be argued that these technical deficiencies render quantum logic incapable of supporting meaningful physical reasoning, and that this provides a justification for viewing the intuitionistic logic of TQT as superior. Unfortunately, this kind of claim cannot be adequately addressed here, but it looks like the most plausible defense of the claim that the intuitionistic logic of TQT represents genuine progress against the orthomodular quantum logic of the Hilbert space formalism.

So, let us suppose for now that this kind of claim is indeed justified, and that the intuitionistic logic of TQT represents a genuine philosophical advance. There are still major interpretational issues that need to be answered regarding $S u b_{c l}(\underline{\Sigma})$. For, recall the argument that lead to the interpretation of $S u b_{c l}(\underline{\Sigma})$ as the lattice of physical propositions. Specifically, we saw that in TQT, we can represent physical propositions by elements of $S u b_{c l}(\underline{\Sigma})$. We did this by taking a physical proposition, associating it with the appropriate 
projection operator $P$, and then taking the daseinisation presheaf $\delta^{o}(P)$ corresponding $P$, which is an element of $S u b_{c l}(\underline{\Sigma})$. Essentially, we began by identifying physical propositions with projection operators (as we do in the Hilbert space formalism), and then defined an injection of the lattice of projection operators into $S u b_{c l}(\underline{\Sigma})$. However, this injection is not surjective. In fact, the image of the assignment that takes each projection operator $P$ to $\delta^{o}(P)$ is only a small subalgebra of $S u b_{c l}(\underline{\Sigma})$. The vast majority of clopen subobjects of the spectral presheaf do not correspond to any projection operator. Now, given that we are supposed to interpret $S u b_{c l}(\underline{\Sigma})$ as the lattice of physical propositions, it seems pertinent to ask what kind of physical propositions these clopen subobjects are supposed to represent. We already know that they cannot possibly represent any proposition of the form $A \in \Delta$, asserting that the value of an observable $A$ lies in a range $\Delta$, since, by the spectral theorem, any such physical proposition will correspond to a particular projection operator $P$, and so will be represented by $\delta^{o}(P)$. So, the task of interpreting the vast majority of the elements of $S u b_{c l}(\underline{\Sigma})$ looks like a difficult one. The elements of this algebra are supposed to represent physical propositions, but most of them do not seem to represent any kind of proposition that is actually used in physics.

For the purposes of illustration, let's consider an example. First, note that the daseinisation operation does not preserve meets . To see this, let $P \in P(H)$ and $V \in V(H)$ be such that $P \notin V$. Then we know that $\frac{\delta^{o}(P)_{V}}{\not \neq P}$ and $\frac{\delta^{o}(1-P)_{V}}{2} \gtrless 1-P$. So $\delta^{o}(P)_{V} \wedge \delta^{o}(1-P)_{V} \neq 0$. But of course, $P \wedge(1-P)=0$ and $\overline{\delta^{o}(P \wedge(1-P))_{V}}=0$. So $\frac{\delta^{o}(P \wedge(1-P))}{S o, \log (P)} \wedge \underline{\delta^{o}(1-P)}$. So, let $P, Q \in P(H)$ be such that $\delta^{o}(P) \wedge \delta^{o}(Q) \neq \delta^{o}(\overline{P \wedge Q) \text {. In }}$ this case, TQT can easily account for the physical significance of $\delta^{o}(P \wedge Q)$. Specifically, we interpret this object as TQT's representation of the physical proposition that is the conjunction of the physical propositions that correspond to $P$ and $Q$ in the Hilbert space formalism. This is unproblematic. However, the same cannot be said for $\delta^{o}(P) \wedge \delta^{o}(Q)$. For, this object cannot be taken to represent the conjunction of the physical propositions that correspond to $P$ and $Q$. This role is already filled by $\delta^{o}(P \wedge Q)$, which is a completely distinct entity. So we need a new physical interpretation for $\delta^{o}(P) \wedge \delta^{o}(Q)$. But it is very difficult to see what kind of satisfactory interpretation we can possibly give in this case. In particular, it can be shown ${ }^{9}$, that there cannot exist any other projection $R$ such that

\footnotetext{
${ }^{9}$ We can argue in the following way: First, define the map $\varepsilon: S u b_{c l}(\Sigma) \rightarrow P(H)$ by $\varepsilon(\underline{S})=$ $\bigvee\left\{P \in P(H) \mid \delta^{o}(P) \leq \underline{S}\right\}$ (this definition and the proof of the properties of $\varepsilon$ can be found in unpublished work by Döring and Cannon). $\varepsilon$ has the important properties that (i) It preserves meets, (ii) $\varepsilon\left(\delta^{o}(P)\right)=P$, for any $P \in P(H)$. Now, if $R$ is such that $\delta^{o}(R)=\delta^{o}(P) \wedge \delta^{o}(Q)$, then, using (i) and (ii) we obtain $R=\varepsilon\left(\underline{\delta^{o}(R)}\right)=\varepsilon\left(\underline{\delta^{o}(P)} \wedge \underline{\left.\delta^{o}(Q)\right)}=\overline{\varepsilon\left(\underline{\delta^{o}}(P)\right.}\right) \wedge \varepsilon\left(\underline{\left.\delta^{o}(\bar{Q})\right)}=\right.$
} 
$\delta^{o}(P) \wedge \delta^{o}(Q)=\delta^{o}(R)$. So if we are to interpret $\delta^{o}(P) \wedge \delta^{o}(Q)$ as a physical proposition, it will have to be an entirely new kind of physical proposition that cannot be represented by any single projection operator.

This looks like a major problem. For, even if intuitionistic logic does turn out to be philosophically preferable to orthomodular quantum logic, it looks like this advantage would have been bought at the cost of introducing a whole new class of phantom propositions that have no natural physical interpretation. Until such an interpretation has been provided, the purported philosophical benefits of the intuitionistic logic of TQT look to have been bought at an unreasonably high cost, i.e. the introduction of a class of physical propositions with no actual physical significance.

\section{Neo-Realism}

In this section, we will draw together the analyses of the preceeding sections in order to assess the relationship of TQT to the realist criteria imposed by Isham and Döring.

Recall that the first realist criterion, PROP, is not generally satisfied by orthodox quantum mechanics, where the Kochen-Specker theorem problematizes the notion of a 'property' of a quantum system. However, in TQT, this criterion becomes less problematic. For, we have seen that, in TQT, it is always possible to assign truth values (in $S E T^{V(H)^{o p}}$ ) simultaneously to all physical propositions associated with a quantum system without violating any algebraic relationships holding between those propositions. In this sense, the notion of a 'property of the system' is always meaningful in TQT. For, if you give me a physical property and a state-vector (or a density matrix), I can always give you a meaningful answer about whether or not the system in question has that physical property. Specifically, I can tell you the truth value of the physical proposition asserting that the system in question has that property. In this sense, TQT satisfies PROP.

Clearly, the notion of a 'property of the system' being employed here differs from the usual notion in a fundamental sense. In TQT, we cannot simply state whether or not the system in question has a given physical property. This is reflected in the complexity of the truth values assigned to physical propositions. Of course, this means that the notion of a 'physical property' loses much of its usual metaphysical weight, and there will be many who are dissatisfied with the apparent lack of any metaphysically substantial notion of a physical property in TQT. One possible response to this concern is to argue

$P \wedge Q$, and so $\delta^{o}(P \wedge Q)=\delta^{o}(R)=\delta^{o}(P) \wedge \delta^{o}(Q)$, which contradicts our original assumption

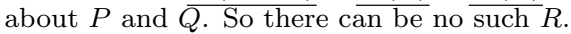


that the notion of a physical property is actually just a by-product of the notion of a physical proposition. Specifically, one could argue that to attribute a physical property to a system is equivalent to asserting one of the physical propositions associated with that system, and that by assigning any extra philosophical weight to the notion of a physical property, one is really just introducing unnecessary metaphysical baggage into their physical theories. This argument then reduces the problem of interpreting the notion of a physical property in TQT to the philosophically simpler problem of interpreting the truth values of TQT, which was addressed in the previous section. At any rate, it seems that the advocate of TQT can plausibly claim that the theory satisfies PROP, given a metaphysically thin notion of 'physical property'.

Isham and Döring's second criterion, BOOLE, was that the physical propositions of the theory should obey classical logic, i.e. should form a Boolean algebra. This criterion is not generally satisfied by TQT. Theorem 2.6.6 only tells us that the physical propositions in TQT form a Heyting algebra, i.e. they obey intuitionistic logic. Thus, TQT does not qualify as a realist theory, under the definition offered by Isham and Doering. Furthermore, we saw that, in obtaining the Heyting algebraic structure of the lattice of physical propositions, TQT introduced a class of purportedly physical propositions that do not have any natural physical interpretation. So, not only does TQT fail to satisfy BOOLE, it also introduces a serious new interpretational difficulty into the logical structure of quantum theory.

So overall, TQT fails to satisfy Isham and Döring's criteria for realist physical theories. Specifically, it fails to satisfy BOOLE and, as we saw in section three, the third criterion cannot be unproblematically applied to it. However, we have seen that TQT does possess the following properties:

(PROP) The idea of 'a property of the system' (i.e. 'the value of a physical quantity') is meaningful, and representable in the theory.

(HEYT) Propositions about the system are handled using intuitionistic logic.

(TRUTH) It is always possible to assign determinate truth-values to all of the physical propositions associated with a system in a consistent way

Now, it is clear that HEYT and TRUTH represent approximations to BOOLE and STATE. The difference between TRUTH and STATE is that TRUTH does not invoke any notion of a physical state, which, as we have seen, is problematic in TQT. In light of the fact that TQT satisfies these weaker forms of the criteria for realist physical theories, it is sometimes described as a 'neo-realist theory'. 
We are now in a position to assess the philosophical significance of the 'neo-realism' offered by TQT. One intuitive way of describing what it means for a physical theory to be realist is to say that realist theories tell us 'how things are' with respect to the systems they describe. So one might be in inclined to ask 'does TQT tell us how things are with quantum systems?'. But this is not an easy question to answer. For, there are multiple possible answers that all, at first blush, appear to have an element of truth about them. Firstly, one could reply that, since TQT satisfies TRUTH, it certainly does tell us how things are with respect to the systems it describes. For, given any physical proposition that one could want to assert of a system described by TQT, the theory will return a definite truth value. On the other hand, one could reply that, since TQT appears to have significant interpretational problems concerning quantum states and state spaces, it does not tell us 'how things are' with respect to the systems it describes, since what this really amounts to is specifying the states of the systems in question. Both of these replies are well motivated, but by different intuitions concerning what it means for a theory to be realist.

The advocate of TQT will likely be inclined to argue that what really matters when we are considering whether a theory 'tells us how things are' with respect to the systems it describes is whether or not the theory satisfies TRUTH. In this respect, TQT's realism has something of a pragmatist flavour. It is concerned primarily with being able to evaluate what we can say about the values of the physical quantities associated with the systems it describes. TQT achieves this, but at a price. For, as we have seen, TQT introduces new interpretational issues of its own. Specifically, it introduces a completely new kind of quantum state space without providing a corresponding new kind of individual quantum state. Thus, TQT does satisfy STATE ("There is a space of microstates such that specifying a microstate leads to unequivocal truth values for all propositions about the system") if we take the 'space of microstates' to be the original Hilbert space of the relevant system, and not the spectral presheaf. But this contradicts one of the basic interpretational assumptions of TQT, that the spectral presheaf plays the role of the quantum state space.

So, although TQT satisfies PROP and TRUTH, it's unclear whether or not it really lives up to the philosophical ideals associated with realist scientific theories. Indeed, it could be argued that TQT even undermines traditional realist metaphysical notions like 'property' and 'state', by focusing primarily on the more pragmatic (and technically complicated) matter of assigning topos theoretic truth values to physical propositions. Furthermore, while it could reasonably be argued that TQT succeeds in constructing a quantum logic that is more amenable to realism, we have seen that this new logic is bought 
at the price of introducing a whole new class of uninterpreted (and apparently uninterpretable) physical propositions.

This all leads naturally to the conclusion that the distinction between realist and non-realist physical theories is actually rather crude and, ultimately, for the case of TQT at least, unhelpful. This is somewhat ironic in so far a TQT is commonly advertised as a kind of realist reformulation of quantum mechanics. But the preceding discussion seems to show that realism is not an absolute notion. It admits of various degrees and dimensions.

\subsection{The covariant approach}

What has been referred to so far as 'topos quantum theory' (TQT) is actually only one of several attempts to use topos theory to gain new insight into the mathematical and conceptual structure of quantum theory (see e.g Heunen et al [2009], Wolters [2013], Adelman and Corbett [1995]). In particular, there is one other approach, commonly referred to in the literature as the 'covariant approach' (because of its use of co-variant, as opposed to contravariant functors), that is very closely related both technically and conceptually to TQT. Indeed, the covariant approach drew much of its inspiration from TQT, and was developed over the past few years by Chris Heunen, Bas Spitters and N.P Landsman, amongst others. Although we cannot give any kind of detailed account of the subtleties of the covariant approach here (for a full formal development and conceptual discussion, see Heunen et al [2009], and for a detailed technical comparison of the covariant approach and TQT, see Wolters [2013]), it is worthwhile to give some preliminary comments regarding the applicability of the arguments offered in this paper to the covariant approach.

In section 2, we noticed that TQT could be seen as a kind of formal embodiment of the Bohrian principle of complementarity (PC). In the covariant approach, the centrality of $\mathrm{PC}$ is made very explicit, and Bohr is often cited as the primary philosophical influence of the project. Thus, we read

'Niels Bohr's "doctrine of classical concepts" states that we can only look at the quantum world through classical glasses, measurement merely providing a 'classical snapshot of reality'. The combination of all such snapshots should then provide a complete picture... This doctrine has a transparent formulation in algebraic quantum theory, to the effect that the empirical content of a quantum theory described by a certain noncommutative $C^{*}$-algebra $A$ is contained in suitable commutative $C^{*}$-algebras associated to $A . '$ (Heunen et al [2009])

Conceptually, the idea is fundamentally the same as in TQT. We formalise PC by somehow 'breaking up' a non-commutative al- 
gebra of observables, corresponding to a quantum system, into its constituent commutative parts, which represent the various classical perspectives that one can take on the system. The fundamental technical difference is that, rather than considering the Von-Neumann algebraic structure of the operator algebras, the covariant approach focuses on the $C^{*}$ algebraic structure. As in TQT, topos theory is the tool that is used to form the 'combination of all such snapshots' that 'provide a complete picture'. To be precise, in the covariant approach, we form the poset $C(A)$ of commutative $C^{*}$ subaglebras of the non-commutative algebra $A$, and then define a covariant functor from $C(A)$ into SETS that takes each element of $C(A)$ to its Gelfand spectrum, and this functor is interpreted as the quantum state space. Clearly, this functor is directly analogous to the spectral presheaf in TQT, and so is denoted by $\underline{\Sigma}$. Just as TQT works in the topos of contravariant set-valued functors over $V(H)$, the covariant approach works in the topos of covariant set-valued functors over $C(A)$. So, in a similar way, it uses the internal logic (which is intuitionistic) and the internal truth-values of the topos.

Now, it turns out that the relevant features of TQT that have been discussed in this paper are mainly all reproduced in the covariant approach, i.e.

(1) KST tells us that $\underline{\Sigma}$ has no global elements, which makes the interpretation of the global elements of $\underline{\Sigma}$ as the states of the covariant approach problematic.

(2) In the covariant approach, the lattice of physical propositions is represented by the lattice of 'open subobjects' of $\Sigma$, and there is a non-surjective injection of the lattice of projections in $A$ into this lattice of open subobjects, analogous to the daseinisation map. Also, the lattice of open subobjects forms a Heyting algebra.

(3) In the covariant approach, individual states are represented by probability measures on $\underline{\Sigma}$. It was shown (see theorem 14 of Heunen et al [2009]) that such probability measures are in bijective correspondence with the quasi states on $A$, which are standardly used to represent quantum states in the $C^{*}$ algebraic approach to quantum theory.

It seems that properties (1)-(3) mean that the covariant approach is subject to the same arguments that have been made about TQT in this paper. Specifically, since the covariant approach represents quantum states in the same way as an existing formalisation (the $C^{*}$ algebraic formalism), which makes no reference to $\underline{\Sigma}$, it seems pertinent to ask in what sense $\underline{\Sigma}$ is really playing the role of a quantum state space. Also, since the logic of the covariant approach is intuitionistic, not classical, the philosophical advantages of the new logical structure will be doubted by many. Finally, the covariant approach is subject to the same criticism that was made of TQT in 
section 4, i.e. it seems to introduce a new class of physical propositions that do not correspond to projection operators without supplying them with a natural physical interpretation. So, in terms of providing a realist reformulation of quantum theory, it seems that the covariant approach is in a very similar situation to TQT.

\section{Conclusion}

In conclusion, we have seen that although TQT fails to satisfy the criteria for realist theories posited by Döring and Isham, the theory does approximate to realism in various key respects. Crucially, the theory does possess a logical structure that allows truth-values to be assigned to all physical propositions in an unproblematic way. In section 4, we argued that the truth-values used in TQT are physically significant, and philosophically preferable to the kinds of truth values that are standardly used in many-valued logical approaches to quantum theory. However, it was also argued that the problem of interpreting those clopen subobjects of the spectral presheaf that are not obtained by the daseinisation of projection operators is a pressing philosophical obstacle for the proponent of TQT, and that before a satisfactory solution to this problem is given, the purported philosophical benefits of TQT's new logical structure seem shallow.

In section 3, it was argued that the identification of the spectral presheaf as the quantum state space of TQT is unjustified, and showed that there is currently no single formal representation of the quantum state-space in TQT that has a satisfactory relationship to the rest of the formalism (in particular, to the representations of individual quantum states in TQT). In section 5 , we saw that analogous conclusions are likely to hold with respect to the covariant approach to quantum theory, due to the structural similarities of that approach with TQT.

Overall, we have seen that to describe TQT as a 'realist' reformulation of quantum theory is actually quite misleading insofar as the theory lacks some of the strong metaphysical properties that are usually associated with realist physical theories. However, we have also seen that the theory overcomes some of the most problematic 'non-realist' properties of orthodox quantum theory in an intuitive and elegant way, and thereby challenges the notion of what kind of realism we should expect from our scientific theories.

\section{Acknowledgements}

Many thanks to the U.K Arts and Humanities Research Council, whose funding supported the author during the writing of this paper. Special thanks also to Richard Pettigrew and the annonymous 
referees for their helpful and patient comments on earlier versions of this paper. Finally, thanks to the British Society for the Philosophy of Science, who gave the author the opportunity to present a draft of this paper at their 2014 annual conference, and to the audience at that conference for their insightful questions.

University of Bristol, Department of Philosophy, Cotham House, Bristol, BS6 6JL, UK

be0367@bristol.ac.uk

\section{References}

[1] Adelman M. and Corbett J.V. [1995]: 'A Sheaf Model for Intuitionistic Quantum Mechanics', Applied Categorical Structures, 3 (1), pp 79-104

[2] Bub, J. [1999]: Interpreting the Quantum World, Cambridge University Press, Revised Edition

[3] Butterfield J. [1999]: 'Topos Theory as a Framework for Partial Truth', in The Scope of Logic, Methodology and Philosophy of Science, Springer, Synthese Library, Vol 315, pp 307-330

[4] Döring A. [2010]: 'Topos Quantum Logic and Mixed States', in B. Coecke, P. Panangaden, P. Selinger (eds), Electronic Notes in Theoretical Computer Science (6th Workshop on Quantum Physics and Logic, QPL VI)

[5] Döring, A. [2011]: 'The Physical Interpretation of Daseinisation', in H Halvorson (ed), Deep Beauty, Cambridge University Press, pp 207-239

[6] Döring A. [2012]: 'Topos-Based Logic for Quantum Systems and Bi-Heyting Algebras', 〈arxiv.org/abs/1202.2750〉

[7] Döring A. and Isham C. [2008]: 'A Topos Foundation for Theories of Physics 1: Formal languages for Physics', Journal of Mathematical Physics, 49, 053515

[8] Döring A. and Isham C. [2011]: ' 'What is a Thing?': Topos Theory in the Foundations of Physics' In New Structures for Physics, B Coecke (ed), Springer, Springer Lecture Notes in Physics, 813, 753-940

[9] Döring, A. and Isham, C. [2012]: 'Classical and Quantum Probabilities as Truth Values', Journal of Mathematical Physics, 53, 032101

[10] Dummett M. [1978]: Truth and Other Enigmas, Harvard University Press, 3rd edition 
[11] Flori C. [2013]: A First Course in Topos Quantum Theory, Springer, Lecture Notes in Physics, Vol 868

[12] Gibbins P. [1987]: Particles and Paradoxes: The Limits of Quantum Logic, Cambridge University Press

[13] Heunen C. , Landsman K. and Spitters B. [2009]: 'A Topos for Algebraic Quantum Theory', Communications in Mathematical Physics, 291 (1), pp 63-110

[14] Isham C. [1997]: 'Topos Theory and Consistent Histories: The Internal Logic of the Set of all Consistent Sets', International Journal of Theoretical Physics, 36: 785-814,

[15] Isham, C. [2011]: 'Topos Methods in the Foundations of Physics', in H Halvorson (ed), Deep Beauty, Cambridge University Press, pp 187-207

[16] Isham C. Butterfield J. [1998]: 'A topos perspective on the Kochen-Specker theorem 1: Quantum States as Generalized Valuations', 〈arxiv.org/abs/quant - ph/9803055〉

[17] Kamlah A. [1981]: 'The Connection Between Reichenbach's Three Valued and Von-Neumann's Lattice Theoretic Quantum Logic', Erkenntniss, 16 (3), pp 315-325

[18] Malinowski J. [1990]: 'The Deduction Theorem for Quantum Logic - Some Negative Results', The Journal of Symbolic Logic, 55 (2), pp 615-625

[19] Wolters S [2013]: 'A Comparison of Two Topos-Theoretic Approaches to Quantum Theory', Communications in Mathematical Physics, 317(1), pp 3-53 